\title{
A Wiener-Tauberian Type Theorem for Arbitrary Normed Algebras with an Approximate Identity
}

\author{
Tobias BLENDEK
}

Helmut Schmidt University Hamburg

(Communicated by K. Takemura)

\begin{abstract}
For an arbitrary normed algebra with an approximate identity, we introduce some notions of invertibility of a net in this algebra with respect to the given approximate identity. By means of that, we show a Wiener-Tauberian type theorem in that general situation. An application yields an abstract Wiener-Tauberian theorem for a class of commutative Banach algebras, which implies the classical Wiener-Tauberian theorem for locally compact Abelian groups.
\end{abstract}

\section{Introduction}

The purpose of this paper is to formulate Wiener's Tauberian theorem from abstract harmonic analysis within the general situation of an arbitrary normed algebra with an approximate identity, see also [2]. In order to repeat the classical case, let $G$ be a locally compact Abelian group with its dual group $\Gamma$ of $G$. The set of all Fourier transforms $\hat{f}$ of functions $f \in L^{1}(G)$ such that, for all $\gamma \in \Gamma$,

$$
\hat{f}(\gamma):=\int_{G} f(s) \overline{\gamma(s)} d s
$$

is denoted by $A(\Gamma)$, and it is a commutative Banach algebra. Then the following result is known as Wiener's Tauberian theorem, see e.g. [1, Theorem 1.1.3]: If a function $\hat{\psi} \in A(\Gamma)$ never vanishes on $\Gamma$, then $\Gamma$ is $\sigma$-compact, and for every $\hat{\vartheta} \in A(\Gamma)$ and $\varepsilon>0$, there are complex numbers $c_{1}, \ldots, c_{n} \in \mathbf{C}$ as well as $x_{1}, \ldots, x_{n} \in G$ such that

$$
\left\|\hat{\vartheta}(\cdot)-\sum_{i=1}^{n} c_{i} \hat{\psi}(\cdot)\left(\cdot, x_{i}\right)\right\|_{A(\Gamma)}<\varepsilon .
$$

Received February 25, 2013; revised June 3, 2013

2010 Mathematics Subject Classification: 46H05 (Primary), 43A25, 43A45, 46H10, 46J20 (Secondary)

Key words and phrases: Normed algebra, approximate identity, Fourier transform, Wiener's Tauberian theorem, commutative Banach algebra 
In the following, let $A$ be an arbitrary normed algebra, and let $(\Lambda, \leq)$ be a directed index set. Then a net $\left\{e_{\lambda}\right\}_{\lambda \in \Lambda}$ in $A$ is called a left (right) approximate identity if, for all $a \in A$,

$$
\left\|a-e_{\lambda} a\right\|_{A} \rightarrow 0 \quad\left(\left\|a-a e_{\lambda}\right\|_{A} \rightarrow 0\right) .
$$

A net $\left\{e_{\lambda}\right\}_{\lambda \in \Lambda}$ is called a two-sided approximate identity if $\left\{e_{\lambda}\right\}_{\lambda \in \Lambda}$ is both a left and a right approximate identity. Furthermore, $\left\{e_{\lambda}\right\}_{\lambda \in \Lambda}$ is called bounded if there is a constant $K>0$ such that $\left\|e_{\lambda}\right\|_{A} \leq K$ for all $\lambda \in \Lambda$. A left (right, two-sided) approximate identity $\left\{e_{\lambda}\right\}_{\lambda \in \Lambda}$ is called sequential if $\Lambda=\mathbf{N}$.

Now, regarding [3, Proposition 12.2], we replace the concept of $\sigma$-compactness in [1, Theorem 1.1.3] by the concept of an approximate identity. Whereas in case of $L^{1}(G)$ such an approximate identity can be constructed, we have to assume its existence in an arbitrary normed algebra. The condition in [1, Theorem 1.1.3], i.e. that a given $\hat{\psi} \in A(\Gamma)$ never vanishes on $\Gamma$, leads us to our notions of invertibility of a net in that normed algebra with respect to the given approximate identity, which yields the announced Wiener-Tauberian type theorem. In fact, we show that to each $\hat{\psi} \in A(\Gamma)$ never vanishing on $\Gamma$, there corresponds such a net in $A(\Gamma)$.

Moreover, we can apply our Wiener-Tauberian type theorem for arbitrary normed algebras with an approximate identity to the situation of completely regular, semi-simple, commutative Banach algebras $A$ with an approximate identity $\left\{e_{\lambda}\right\}_{\lambda \in \Lambda}$ in $A$ such that each Gelfand transform $\widehat{e_{\lambda}}$ of $e_{\lambda}$ has compact support in the maximal ideal space $\Phi_{A}$ of $A$. For definitions, we refer to [4]. More precisely, we get an abstract Wiener-Tauberian theorem by proving that the principal ideal $a A:=\{a x: x \in A\}$ generated by $a \in A$ is dense in $A$ if the Gelfand transform $\hat{a}$ of $a$ never vanishes on the maximal ideal space $\Phi_{A}$ of $A$. As a consequence, we obtain the classical Wiener-Tauberian theorem for $A(\Gamma)$ again.

\section{A Wiener-Tauberian Type Theorem}

DEFINITION 2.1. Let $A$ be a normed algebra with a left (right) approximate identity $\left\{e_{\lambda}\right\}_{\lambda \in \Lambda}$ in $A$.

(a) We call a net $\left\{a_{\lambda}\right\}_{\lambda \in \Lambda}$ in $A$ right (left) invertible in $A$ if there is a net $\left\{b_{\lambda}\right\}_{\lambda \in \Lambda}$ in $A$ such that, for all $\lambda \in \Lambda$,

$$
a_{\lambda} b_{\lambda}=e_{\lambda} \quad\left(b_{\lambda} a_{\lambda}=e_{\lambda}\right) .
$$

If $\left\{e_{\lambda}\right\}_{\lambda \in \Lambda}$ is a two-sided approximate identity in $A$, we say that $\left\{a_{\lambda}\right\}_{\lambda \in \Lambda}$ is invertible in $A$ if it is both left and right invertible.

(b) We call a net $\left\{a_{\lambda}\right\}_{\lambda \in \Lambda}$ in $A$ right-extended (left-extended) invertible in $A$ if there is a net $\left\{b_{\lambda}\right\}_{\lambda \in \Lambda}$ in $A$ such that, for all $\lambda \in \Lambda$,

$$
a_{\lambda} b_{\lambda} e_{\lambda}=e_{\lambda} \quad\left(e_{\lambda} b_{\lambda} a_{\lambda}=e_{\lambda}\right) .
$$

If $\left\{e_{\lambda}\right\}_{\lambda \in \Lambda}$ is a two-sided approximate identity in $A$, we say that $\left\{a_{\lambda}\right\}_{\lambda \in \Lambda}$ is extended invertible in $A$ if it is both left-extended and right-extended invertible. 
(c) We call a net $\left\{a_{\lambda}\right\}_{\lambda \in \Lambda}$ in $A$ right-centered (left-centered) invertible in $A$ if there is a net $\left\{b_{\lambda}\right\}_{\lambda \in \Lambda}$ in $A$ such that, for all $\lambda \in \Lambda$,

$$
a_{\lambda} e_{\lambda} b_{\lambda}=e_{\lambda} \quad\left(b_{\lambda} e_{\lambda} a_{\lambda}=e_{\lambda}\right) .
$$

If $\left\{e_{\lambda}\right\}_{\lambda \in \Lambda}$ is a two-sided approximate identity in $A$, we say that $\left\{a_{\lambda}\right\}_{\lambda \in \Lambda}$ is centered invertible in $A$ if it is both left-centered and right-centered invertible.

THEOREM 2.2. Let A be a normed algebra with a left (right) approximate identity $\left\{e_{\lambda}\right\}_{\lambda \in \Lambda}$ in $A$. Let $\left\{a_{\lambda}\right\}_{\lambda \in \Lambda}$ be a net in $A$ such that it is

(a) right (left) invertible in $A$ or

(b) right-extended (left-extended) invertible in A or

(c) right-centered (left-centered) invertible in A.

Then, in each of these cases, for every $c \in A$, there is a net $\left\{f_{\lambda}\right\}_{\lambda \in \Lambda}$ in A such that

$$
\left\|c-a_{\lambda} f_{\lambda}\right\|_{A} \rightarrow 0 \quad\left(\left\|c-f_{\lambda} a_{\lambda}\right\|_{A} \rightarrow 0\right) .
$$

Proof. (a) Let $\left\{a_{\lambda}\right\}_{\lambda \in \Lambda}$ be a right (left) invertible net in $A$. Then there is a net $\left\{b_{\lambda}\right\}_{\lambda \in \Lambda}$ in $A$ such that $a_{\lambda} b_{\lambda}=e_{\lambda}\left(b_{\lambda} a_{\lambda}=e_{\lambda}\right)$ for all $\lambda \in \Lambda$. Let $c$ be an arbitrary element of $A$. For each $\lambda \in \Lambda$, we set

$$
f_{\lambda}:=b_{\lambda} c \in A \quad\left(f_{\lambda}:=c b_{\lambda} \in A\right)
$$

Hence, we get

$$
\begin{aligned}
\left\|c-a_{\lambda} f_{\lambda}\right\|_{A} & =\left\|c-a_{\lambda} b_{\lambda} c\right\|_{A}=\left\|c-e_{\lambda} c\right\|_{A} \rightarrow 0 \\
\left(\left\|c-f_{\lambda} a_{\lambda}\right\|_{A}\right. & \left.=\left\|c-c b_{\lambda} a_{\lambda}\right\|_{A}=\left\|c-c e_{\lambda}\right\|_{A} \rightarrow 0\right) .
\end{aligned}
$$

(b) Similar to $(a)$, the assertion follows by setting, for each $\lambda \in \Lambda$,

$$
f_{\lambda}:=b_{\lambda} e_{\lambda} c \in A \quad\left(f_{\lambda}:=c e_{\lambda} b_{\lambda} \in A\right) .
$$

(c) Similar to (a), the assertion follows by setting, for each $\lambda \in \Lambda$,

$$
f_{\lambda}:=e_{\lambda} b_{\lambda} c \in A \quad\left(f_{\lambda}:=c b_{\lambda} e_{\lambda} \in A\right) .
$$

DEFINITION 2.3. Let $A$ be a normed algebra with a bounded two-sided approximate identity $\left\{e_{\lambda}\right\}_{\lambda \in \Lambda}$ in $A$. We call a net $\left\{a_{\lambda}\right\}_{\lambda \in \Lambda}$ in A quadratically invertible in $A$ if there is a net $\left\{b_{\lambda}\right\}_{\lambda \in \Lambda}$ in $A$ such that, for all $\lambda \in \Lambda$,

$$
a_{\lambda} e_{\lambda} b_{\lambda} e_{\lambda}=e_{\lambda}^{2}
$$

THEOREM 2.4. Let $A$ be a normed algebra with a bounded two-sided approximate identity $\left\{e_{\lambda}\right\}_{\lambda \in \Lambda}$ in $A$. If $\left\{a_{\lambda}\right\}_{\lambda \in \Lambda}$ is a quadratically invertible net in $A$, then for every $c \in A$, there is a net $\left\{f_{\lambda}\right\}_{\lambda \in \Lambda}$ in A such that

$$
\left\|c-a_{\lambda} f_{\lambda}\right\|_{A} \rightarrow 0 .
$$


PROOF. Let $\left\{a_{\lambda}\right\}_{\lambda \in \Lambda}$ be a quadratically invertible net in $A$. Then there is a net $\left\{b_{\lambda}\right\}_{\lambda \in \Lambda}$ in $A$ such that $a_{\lambda} e_{\lambda} b_{\lambda} e_{\lambda}=e_{\lambda}^{2}$ for all $\lambda \in \Lambda$. Let $c$ be an arbitrary element of $A$. For each $\lambda \in \Lambda$, we set

$$
f_{\lambda}:=e_{\lambda} b_{\lambda} e_{\lambda} c e_{\lambda} \in A .
$$

Hence, since the net $\left\{e_{\lambda}^{2}\right\}_{\lambda \in \Lambda}$ is a bounded two-sided approximate identity in $A$, too, we get

$$
\begin{aligned}
\left\|c-a_{\lambda} f_{\lambda}\right\|_{A} & \leq\left\|c-c e_{\lambda}\right\|_{A}+\left\|c e_{\lambda}-a_{\lambda} f_{\lambda}\right\|_{A} \\
& =\left\|c-c e_{\lambda}\right\|_{A}+\left\|c e_{\lambda}-\left(a_{\lambda} e_{\lambda} b_{\lambda} e_{\lambda}\right) c e_{\lambda}\right\|_{A} \\
& =\left\|c-c e_{\lambda}\right\|_{A}+\left\|\left(c-e_{\lambda}^{2} c\right) e_{\lambda}\right\|_{A} \\
& \leq\left\|c-c e_{\lambda}\right\|_{A}+\left\|c-e_{\lambda}^{2} c\right\|_{A}\left\|e_{\lambda}\right\|_{A} \\
& \leq\left\|c-c e_{\lambda}\right\|_{A}+K\left\|c-e_{\lambda}^{2} c\right\|_{A} \\
& \rightarrow 0 .
\end{aligned}
$$

COROLLARY 2.5. Let A be a normed algebra with a bounded two-sided approximate identity (left approximate identity) $\left\{e_{\lambda}\right\}_{\lambda \in \Lambda}$ in $A$, and let I be a closed two-sided ideal in A. In addition, one of the following two conditions should hold:

(a) There is a net $\left\{a_{\lambda}\right\}_{\lambda \in \Lambda}$ in A such that it is quadratically invertible (right invertible, right-extended invertible, right-centered invertible) in $I$.

(b) There is a net $\left\{a_{\lambda}\right\}_{\lambda \in \Lambda}$ in I such that it is quadratically invertible (right invertible, right-extended invertible, right-centered invertible) in $A$.

Then we have $I=A$.

PROOF. (a) If $\left\{a_{\lambda}\right\}_{\lambda \in \Lambda}$ is a net in $A$ such that it is quadratically invertible (right invertible, right-extended invertible, right-centered invertible) in $I$, then, according to Theorem 2.4 (Theorem 2.2(a), (b), (c)), for every $c \in A$, there is a net $\left\{f_{\lambda}\right\}_{\lambda \in \Lambda}$ in $I$ such that

$$
\left\|c-a_{\lambda} f_{\lambda}\right\|_{A} \rightarrow 0 .
$$

Since $I$ is a two-sided ideal in $A$, we have $a_{\lambda} f_{\lambda} \in I$ for all $\lambda \in \Lambda$. Since $I$ is closed, we conclude that $c \in I$, i.e. we get $I=A$.

(b) This assertion follows in like manner as $(a)$.

An application of Theorem 2.2(b), (c) and Theorem 2.4, respectively, yields the following Wiener-Tauberian theorem in the classical case of locally compact Abelian groups.

REMARK 2.6. Let $G$ be a locally compact Abelian group with its dual group $\Gamma$ of $G$. If $\hat{\psi} \in A(\Gamma)$ never vanishes on $\Gamma$, then there is a sequential, bounded approximate identity $\left\{\widehat{e_{n}}\right\}_{n \in \mathbf{N}}$ in $A(\Gamma)$ such that for every $\hat{\vartheta} \in A(\Gamma)$ and $\varepsilon>0$, there are an $N(\varepsilon)$ and a sequence 
$\left\{\widehat{f}_{n}\right\}_{n \in \mathbf{N}}$ in $A(\Gamma)$ such that, for all $n \geq N(\varepsilon)$,

$$
\left\|\hat{\vartheta}-\hat{\psi} \widehat{f}_{n}\right\|_{A(\Gamma)}<\varepsilon .
$$

Hence, the principal ideal $\hat{\psi} A(\Gamma):=\{\hat{\psi} \hat{\varphi}: \hat{\varphi} \in A(\Gamma)\}$ generated by $\hat{\psi}$ is dense in $A(\Gamma)$.

ProOF. Let $\hat{\psi} \in A(\Gamma)$ never vanish, i.e. let the zero set $Z(\hat{\psi})$ be empty. Thus we have supp $\hat{\psi}=\Gamma$, where supp $\hat{\psi}$ denotes the support of $\hat{\psi}$. Then, by [1, Proposition 1.1.2(b)], the dual group $\Gamma$ is $\sigma$-compact. Consequently, regarding [1, Theorem 1.2.1], there is a sequential, bounded approximate identity $\left\{\widehat{e}_{n}\right\}_{n \in \mathbf{N}}$ in $A(\Gamma)$ such that, for all $n \in \mathbf{N}$,

$$
\left\|\widehat{e_{n}}\right\|_{A(\Gamma)}=1, \quad \widehat{e_{n}} \geq 0 \quad \text { and } \quad \widehat{e_{n}} \in A_{c}(\Gamma):=A(\Gamma) \cap C_{c}(\Gamma),
$$

where $C_{c}(\Gamma)$ denotes the algebra of all continuous, complex-valued functions on $\Gamma$ with compact support. Let $C_{n}:=\operatorname{supp} \widehat{e_{n}}$ be the compact support of $\widehat{e_{n}}$ for all $n \in \mathbf{N}$. Since $\hat{\psi}(\gamma) \neq 0$ for all $\gamma \in \Gamma$ and since $C_{n} \subseteq \Gamma$ is compact for all $n \in \mathbf{N}$, there is a $\widehat{\psi}_{n}^{\prime} \in A(\Gamma)$ according to Wiener's inversion theorem, see e.g. [1, Proposition 1.1.5(b)], such that, for all $\gamma \in C_{n}$

$$
\widehat{\psi}_{n}^{\prime}(\gamma)=1 / \hat{\psi}(\gamma)
$$

Since $A(\Gamma)$ is a commutative Banach algebra, we conclude that, for all $\gamma \in C_{n}$,

$$
\hat{\psi}(\gamma) \widehat{e_{n}}(\gamma){\widehat{\psi_{n}}}^{\prime}(\gamma)=\hat{\psi}(\gamma){\widehat{\psi_{n}}}^{\prime}(\gamma) \widehat{e_{n}}(\gamma)=\widehat{e_{n}}(\gamma) .
$$

For each $\gamma \notin C_{n}$, we have $\widehat{e_{n}}(\gamma)=0$, and hence, (2.1) is valid for all $n \in \mathbf{N}$ and for all $\gamma \in \Gamma$. Of course, we also have, for all $n \in \mathbf{N}$ and for all $\gamma \in \Gamma$,

$$
\hat{\psi}(\gamma) \widehat{e_{n}}(\gamma){\widehat{\psi_{n}}}^{\prime}(\gamma) \widehat{e_{n}}(\gamma)={\widehat{e_{n}}}^{2}(\gamma) \text {. }
$$

So, identifying $\hat{\psi} \in A(\Gamma)$ with the sequence $\left\{\widehat{\psi_{n}}\right\}_{n \in \mathbf{N}}$ defined by $\widehat{\psi_{n}}:=\hat{\psi}$ for all $n \in \mathbf{N}$, we see that $\hat{\psi}$ is quadratically invertible (extended invertible, centered invertible) with respect to the sequential, bounded approximate identity $\left\{\widehat{e}_{n}\right\}_{n \in \mathbf{N}}$ in $A(\Gamma)$. Consequently, the assertion follows from Theorem 2.4 (Theorem 2.2(b), (c)).

Now, we show an abstract Wiener-Tauberian theorem for a class of commutative Banach algebras by using Theorem $2.2(b),(c)$.

THEOREM 2.7. Let A be a completely regular, semi-simple, commutative Banach algebra with an approximate identity $\left\{e_{\lambda}\right\}_{\lambda \in \Lambda}$ in A such that each Gelfand transform $\widehat{e_{\lambda}}$ of $e_{\lambda}$ has compact support in the maximal ideal space $\Phi_{A}$ of $A$. Let a be an arbitrary element of A. If $\hat{a}$ never vanishes on $\Phi_{A}$, then the principal ideal $a A:=\{a x: x \in A\}$ generated by $a$ is dense in $A$.

Proof. Assume that $\hat{a}$ never vanishes on $\Phi_{A}$ and take $\lambda \in \Lambda$ arbitrarily. Denote by $K_{\lambda}$ the compact support of $\widehat{e_{\lambda}}$. By hypothesis, $|\hat{a}(\varphi)| \geq \delta_{\lambda}$ holds for all $\varphi \in K_{\lambda}$ and some $\delta_{\lambda}>0$. Then, according to [4, Theorem 3.6.15] and [4, Theorem 3.7.1], we can find a $b_{\lambda} \in A$ such 
that $\hat{a}(\varphi) \widehat{b_{\lambda}}(\varphi)=1$ for all $\varphi \in K_{\lambda}$. Therefore, we obtain $\hat{a} \widehat{b_{\lambda}} \widehat{e_{\lambda}}=\widehat{e_{\lambda}}$, and hence $a b_{\lambda} e_{\lambda}=e_{\lambda}$ since $A$ is semi-simple. Put $a_{\lambda}:=a$ for each $\lambda \in \Lambda$. Then $\left\{a_{\lambda}\right\}_{\lambda \in \Lambda}$ is an extended invertible and, of course, a centered invertible net in $A$ with respect to $\left\{e_{\lambda}\right\}_{\lambda \in \Lambda}$. Consequently, it follows from Theorem 2.2(b), (c) that the principal ideal $a A$ is dense in $A$.

REMARK 2.8. (a) Of course, we also have the converse of Theorem 2.7: Let $A$ be a commutative Banach algebra with maximal ideal space $\Phi_{A}$, and let $a$ be an arbitrary element of $A$. If the principal ideal $a A:=\{a x: x \in A\}$ generated by $a$ is dense in $A$, then the Gelfand transform $\hat{a}$ of $a$ never vanishes on $\Phi_{A}$.

(b) Let $G$ be a locally compact Abelian group, and let $\psi \in L^{1}(G)$ such that $\hat{\psi} \in A(\Gamma)$ never vanishes on $\Gamma$. Since $L^{1}(G)$ is a completely regular, semi-simple, commutative Banach algebra with the convolution product $*$ and since, according to [1, Theorem 1.2.1], there is a sequential, bounded approximate identity in $A(\Gamma)$ with compact support in $\Gamma$, it follows from Theorem 2.7 that the principal ideal $\psi * L^{1}(G):=\left\{\psi * \varphi: \varphi \in L^{1}(G)\right\}$ generated by $\psi$ is dense in $L^{1}(G)$, i.e. we obtain the classical Wiener-Tauberian theorem for locally compact Abelian groups, see Remark 2.6.

ACKNOWLEDGMENT. We would like to thank the referee for his useful comments.

\section{References}

[ 1 ] J. J. Benedetto, Spectral Synthesis, Academic Press, Inc., New York, 1975.

[2] T. BlendeK, Normen von Projektionen im Dual einer kompakten Kac-Algebra, Doctoral dissertation, University of Hamburg, Hamburg, 2010.

[ 3 ] R. S. DorAn and J. Wichmann, Approximate Identities and Factorization in Banach Modules, Lecture Notes in Math. 768, Springer-Verlag, Berlin-Heidelberg-New York, 1979.

[ 4 ] C. E. RickART, General Theory of Banach Algebras, D. Van Nostrand Company, Inc., Princeton, New Jersey, 1960.

Present Address:

DePartment of Mathematics And Statistics, HELMUT SCHMIDT UNIVERSITY HAMBURG, HolstenhofWeg 85, 22043 Hamburg, Germany.

e-mail: tobias.blendek@gmx.de 